\title{
Can We Identify Patients with High Risk of Osteoarthritis Progression Who Will Respond to Treatment? A Focus on Biomarkers and Frailty
}

\author{
Nigel Arden ${ }^{1,2} \cdot$ Pascal Richette $^{3,4} \cdot$ Cyrus Cooper $^{1,5} \cdot$ Olivier Bruyère $^{6} \cdot$ Eric Abadie $^{7} \cdot$ Jaime Branco $^{8,9}$. \\ Maria Luisa Brandi ${ }^{10}$ - Francis Berenbaum ${ }^{11,12} \cdot$ Cécile Clerc $^{13}$ - Elaine Dennison ${ }^{4,5} \cdot$ Jean-Pierre Devogelaer $^{14}$. \\ Marc Hochberg $^{15} \cdot$ Pieter D'Hooghe $^{16}$ - Gabriel Herrero-Beaumont ${ }^{17} \cdot$ John A. Kanis $^{18} \cdot$ Andrea Laslop $^{19}$. \\ Véronique Leblanc $^{20}$ - Stefania Maggi ${ }^{21}$ - Giuseppe Mautone ${ }^{22}$ - Jean-Pierre Pelletier ${ }^{23}$ - Florence Petit-Dop ${ }^{24}$. \\ Susanne Reiter-Niesert ${ }^{25}$ - René Rizzoli ${ }^{26}$ - Lucio Rovati $^{27}$ - Eleonora Tajana Messi ${ }^{22}$ - Yannis Tsouderos ${ }^{24}$. \\ Johanne Martel-Pelletier ${ }^{23} \cdot$ Jean-Yves Reginster $^{6}$
}

Published online: 18 June 2015

(C) The Author(s) 2015. This article is published with open access at Springerlink.com

\begin{abstract}
Osteoarthritis (OA), a disease affecting different patient phenotypes, appears as an optimal candidate for personalized healthcare. The aim of the discussions of the European Society for Clinical and Economic Aspects of Osteoporosis and Osteoarthritis (ESCEO) working group was to explore the value of markers of different sources in defining different phenotypes of patients with OA. The ESCEO organized a series of meetings to explore the
\end{abstract}

\section{Olivier Bruyère}

olivier.bruyere@ulg.ac.be

$1 \quad$ NIHR Musculoskeletal Biomedical Research Unit, University of Oxford, Oxford, UK

2 ARUK Sports, Exercise and Osteoarthritis Centre of Excellence, Oxford, UK

3 UFR Médicale, Paris Diderot University, Paris, France

4 AP-HP, Hôpital Lariboisière, Fédération de Rhumatologie, Paris, France

5 MRC Epidemiology Resource Centre, University of Southampton, Southampton, UK

6 Department of Public Health, Epidemiology and Health Economics, University of Liège and CHU Centre Ville, Chu Sart Tilman B23, 4000 Liège, Belgium

7 Division of Cardiology, Ambroise Paré Hospital, Paris, France

8 CEDOC, Department of Rheumatology, Faculdade de Ciências Médicas, Universidade Novade Lisboa, Lisbon, Portugal

9 CHLO, EPE, Hospital Egas Moniz, Lisbon, Portugal

10 Department of Internal Medicine, University of Florence, Florence, Italy

11 Department of Rheumatology, Université Pierre and Marie Curie AP-HP, Hôpital Saint-Antoine, Paris, France possibility of identifying patients who would most benefit from treatment for OA, on the basis of recent data and expert opinion. In the first meeting, patient phenotypes were identified according to the number of affected joints, biomechanical factors, and the presence of lesions in the subchondral bone. In the second meeting, summarized in the present article, the working group explored other markers involved in OA. Profiles of patients may be defined

12 University of Paris, 06-INSERM UMR-S938, Paris, France

13 Laboratoires Genevrier, Antibes, France

14 Department of Rheumatology, Saint-Luc University Hospital, Catholic University, Louvain, Belgium

15 Division of Rheumatology and Clinical Immunology, Department of Medicine, University of Maryland School of Medicine, Geriatric Research, Education and Clinical Center, Maryland VA Health Care System, Baltimore, MD, USA

16 Department of Orthopaedics and Sports Medicine, Aspetar Hospital, Doha, Qatar

17 Bone and Joint Research Unit, Fundación Jiménez Diaz, Madrid, Spain

18 Centre for Metabolic Bone Diseases, University of Sheffield Medical School, Sheffield, UK

19 Scientific Office, Austrian Agency for Health and Food Safety (AGES), Vienna, Austria

20 Expanscience Laboratoires, Courbevoie, France

21 Aging Program, National Research Council, Padua, Italy

22 IBSA Institut Biochimique SA Via del Piano, 6915 Pambio-Noranco, Switzerland

23 Osteoarthritis Research Unit, University of Montreal Hospital Research Centre (CRCHUM), Notre-Dame Hospital, Montreal, QC, Canada 
according to their level of pain, functional limitation, and presence of coexistent chronic conditions including frailty status. A considerable amount of data suggests that magnetic resonance imaging may also assist in delineating different phenotypes of patients with OA. Among multiple biochemical biomarkers identified, none is sufficiently validated and recognized to identify patients who should be treated. Considerable efforts are also being made to identify genetic and epigenetic factors involved in OA, but results are still limited. The many potential biomarkers that could be used as potential stratifiers are promising, but more research is needed to characterize and qualify the existing biomarkers and to identify new candidates.

\section{Key Points}

Osteoarthritis affects different patient phenotypes with heterogeneous clinical presentation, rate of progression, and response to therapy, and thus appears as an optimal candidate for personalized medicine.

The level of pain, functional limitation, and presence of coexistent chronic conditions including frailty status should be considered to guide treatment decisions.

Magnetic resonance imaging-based diagnosis could be used in drug development and in clinical practice to identify patients more likely to benefit from treatment.

Promising potential biomarkers (e.g., biochemical, genetic, epigenetic) currently under investigations could be used in the near future to guide clinical decision making.

\section{Introduction}

As non-communicable diseases increase with the rise in life expectancy and with the changes in lifestyle, the traditional approach to their management should be reconsidered. Current medicine is still largely based on the 'one-size-fits-all' model where patients diagnosed

24 Institut de Recherches Internationales Servier, Suresnes, France

25 Federal Institute for Drugs and Medical Devices (BfArM), Bonn, Germany

26 Service of Bone Diseases, Department of Rehabilitation and Geriatrics, University Hospitals and Faculty of Medicine of Geneva, Geneva, Switzerland

27 Clinical Research Unit, Rottapharm/Madaus, Monza, Italy with the same condition often are prescribed the same medication at the same dose. For some medications, this model potentially can lead to poor response, preventable occurrence of side effects, and increased healthcare costs.

For these reasons, a more patient-centric or personalized approach of medical practice has been proposed and the conventional model is being replaced by new approaches in which the health of the patients can be managed according to their individual biologic or risk factors. The concept of 'stratified medicine' refers to this targeting of treatments (including pharmacological and non-pharmacological interventions) according to the biological or risk characteristics shared by subgroups of patients. The potential to use biomarkers for identifying patients with the greatest chance of benefit and the lowest risk of experiencing adverse events in response to a given therapy is anticipated to have a major effect on both clinical practice (e.g., choice of treatment, dosing, or duration of treatment) and the development of new drugs and diagnostics [1,2].

Osteoarthritis (OA) is a common slowly progressive condition that may affect the structure of all joint tissues, and is a major cause of pain and chronic disability in the elderly [3]. The lack of a universal definition of OA is probably because of the complexity of processes underlying its pathogenesis [4] and to the diversity in clinical presentation, rate of disease progression, pattern of joint involvement, and joint tissue affected [5]. Considering this marked heterogeneity, it appears unlikely that one treatment will benefit all phenotypes of patients. OA thus appears as an optimal candidate for personalized medicine.

The ESCEO (European Society for Clinical and Economic Aspects of Osteoporosis and Osteoarthritis) has previously explored a variety of pivotal topics in OA, including the question of how best to define responders to treatment for drug development [4] and the value of magnetic resonance imaging (MRI) in the diagnosis of the disease and the prediction of the hard outcome of joint replacement surgery [6].

An ESCEO working meeting convened in October 2012 discussed the value of biomarkers currently investigated in drug development in OA with a focus on the potential avenues for future research [7]. In 66 relevant publications, numerous candidate biomarkers from multiple sources were identified. Some were considered promising but none of the best candidates has entered clinical use.

In a meeting held in October 2013, an ESCEO-EUGMS (European Union Geriatric Medicine Society) working group discussed the possibility of identifying patients who would most benefit from treatment for OA, to better orientate research and to identify relevant outcomes in clinical trials. Patient profiles were identified according to the 
number of affected joints, biomechanical factors, and the presence of lesions in the subchondral bone [8].

In this second meeting held in May 2014, the working group explored other markers involved in OA that could be used to define patients to be treated, such as imaging, clinical, biochemical, genetic, and epigenetic markers. This is the subject of the present article.

\section{Process and Outcomes}

As in previous initiatives and publications, the ESCEO working group consisted of clinical scientists and experts in the field of $\mathrm{OA}$ in academia and the pharmaceutical industry, and representatives of national or European licensing authorities giving their contribution on a personal basis. Members of the group were asked to prepare a full review of the literature on imaging markers (JMP), clinicalbiochemical markers $(\mathrm{MH})$, stratified medicine in $\mathrm{OA}$ (CC), genetic-epigenetic markers (ED), and the association of OA with frailty status (SM).

They identified relevant articles, reviews, and abstracts in a search of PubMed/MEDLINE and EMBASE for English language articles published between 1990 and October 2014. The initial search strategy included the terms osteoarthritis, risk factor, predictor, progression, guidelines, biomarkers, MRI, and phenotype. Separate sub-searches were also performed using a cross search of the above terms combined, and additional references were selected from the reference lists of selected articles and the presentations made during the working meeting. The narrative is therefore largely based upon expert opinion. Relevant items were selected by the authors according to their quality and pertinence for discussion by the ESCEO working group. After the presentations given at the working meeting, a comprehensive discussion was held within the group and shared conclusions were reached. Two electronic consultation rounds occurred on the draft manuscript to achieve a final consensus.

\section{Risk Factors for Progression and Predictors of Response to Treatments}

\subsection{Risk Factors for Progression}

Increasing our knowledge regarding the predictors of progression of $\mathrm{OA}$ is important for optimal treatment allocation and clinical investigations of new therapies [9]. Not all patients will progress symptomatically, radiologically, or to joint replacement and treatments need to be focused to those most at need.
A number of risk factors for the progression of OA have been identified including obesity, radiographic features, biomechanical derangement, multi-joint OA, and synovitis/effusion $[10,11]$. In a study on 561 patients, although more than half of the patients had stable Kellgren-Lawrence grades over 15 years, patients with a baseline Kellgren-Lawrence grade 1 were twice as likely to progress as those with a baseline Kellgren-Lawrence grade 0 [12]. A systematic review to identify risk factors for radiographic progression showed that knee malalignment was an independent risk factor for the progression of knee OA [11]. A review of 36 high-quality studies was performed to provide an overview of prognostic factors of knee OA progression [9]. While sex, knee pain, radiologic severity, knee injury, quadriceps strength, and regular sport activities did not seem to be predictive, the best evidence synthesis yielded strong evidence that the serum levels of hyaluronic acid, a marker of synovial inflammation, and generalized OA (i.e., different joints affected at the same time) are predictive for the progression of knee OA [9]. In studies focussing on patient-reported outcomes (pain or activity limitations), as opposed to radiographic progression, prognostic factors that were identified included radiographic, biomechanical, biological, and clinical factors but also psychological and social factors $[13,14]$. Most of these findings need to be validated and interactions between individual potential predictors of progression need to be identified, providing a direction for future research.

\subsection{Predictors of Response to Treatment}

However, predictors for progression of the disease and need for treatment may not be the same as the predictors of response to treatment. Current OA treatment includes nonpharmacological therapies, such as physical therapy, weight loss in obese patients or device-based treatments, and pharmacologic therapies, primarily intended to alleviate pain and improve/preserve functional ability. When all previous modalities have failed, surgery such as joint replacement is the main option. A recent algorithm was developed to advise on the possible stepwise approach to the sequence of interventions [15]. All current treatments are symptom-modifying drugs as there are no licensed treatments that prevent the progression of the disease. Disease-modifying OA drugs (DMOADs) have therefore become a major focus of research, but their development involves a number of difficulties, including slow disease progression and a lack of sensitivity of tools recommended by the regulatory agencies [16]. The current treatments (except for joint replacement) have at best modest albeit clinically relevant efficacies, and are sometimes associated with substantial side effects or costs [17]. Part of this modest efficacy might be explained by the inclusion of 
heterogeneous groups of OA patients in trials. This highlights the need to identify subgroups of patients who might be responsive to current treatments. Furthermore, all treatments have associated side effects, stressing the importance of focusing therapy to patients with the adequate phenotype.

\section{Imaging Markers}

Radiography is the most commonly used technology and is recommended by regulatory agencies for assessing DMOAD. Although radiography has weaknesses, including a poor sensitivity to changes in cartilage width, it is cheap and accessible and has been shown to predict the progression of $\mathrm{OA}$ and response to some intra-articular and oral treatments. The main limitation of plain radiography is its inability to assess other important joint structures such as the synovium, meniscus, and bone marrow lesions (BML). Important progress has been made in the development of MRI technology allowing direct, precise, and reliable assessment of multiple tissues and their changes over time targeting mostly hip and knee OA. MRI has been extensively used in a number of studies assessing cartilage volume loss, BML, synovial membrane thickness, meniscal extrusion, Hoffa's fat pad and, less extensively studied, synovial fluid effusion size.

\subsection{Extent of Cartilage Damage}

A number of studies suggest that MRI may assist in delineating different phenotypes of patients with OA. Some clinical trials have assessed the effect of drugs on articular structural change over time in patients with knee OA including licofelone [18], chondroitin sulfate [19], strontium ranelate [20], and sprifermin [21]. In these trials in patients at a moderate to severe stage of the disease, these agents were shown to have a beneficial effect on cartilage volume loss, which was mostly seen at the lateral compartment. In a study using data from the OsteoArthritis Initiative progression cohort, glucosamine plus chondroitin treatment significantly reduced the cartilage volume loss, predominantly in patients with mild to moderate knee OA, but showed no protective effect in those with already severe disease [22].

This study also suggests that in patients with less extensive cartilage damage, treatment provided protection in both medial and lateral compartments.

\subsection{Presence of Bone Marrow Lesions}

MRI has also been used in a number of studies to select knee OA patients with BML.
A proof-of-concept trial in knee OA patients evaluated the effect of zoledronic acid, a bisphosphonate, on BML change at 1 year. Although data showed a significant reduction in BML size at 6 months, and a numerical trend towards a reduction at 12 months, the cartilage volume and cartilage loss were not assessed [23]. However, other putative DMOADs were tested for their effects on the change over time of both BML in the medial compartment and cartilage volume. Two such drugs were studied, licofelone [18] and strontium ranelate [20]. In general, data showed a strong association for the compounds tested between BML change and cartilage volume loss in the same or related topographical site.

\subsection{Presence of Meniscal Extrusion}

Although the presence of meniscal extrusion has been shown to be associated with greater cartilage volume loss and to be a strong marker of OA progression [23, 24], to our knowledge there has been no clinical trial using MRI investigating the effect on meniscal extrusion of drug response to treatment. However, post hoc analyses of studies exploring the effect of putative DMOADs (licofelone [18], strontium ranelate [25], and chondroitin and glucosamine sulfate [26]) and one study looking at the presence of meniscal extrusion in knee OA patients with neuropathic pain [27], argue in favor of an MRI-based diagnosis of meniscal extrusion in clinical practice. This would help physicians identify knee OA patients who are more likely to benefit from treatment, namely those with mild to moderate OA with meniscal extrusion, or those with more symptomatic disease but without meniscal extrusion. Moreover, co-localized knee structural pathologies such as meniscal extrusion/damage and BML further increased the risk of cartilage loss [25, 28] highlighting that the response to treatment of knee OA patients can be greatly influenced by the presence or absence of multiple risk factors associated with the progression of structural changes.

\section{Clinical and Biochemical Biomarkers}

\subsection{Clinical Biomarkers}

Among useful clinical markers, the most important and obvious are the presence and severity of pain and of functional limitation, which are the primary indications of licensed medications in OA. Demographic factors (age, sex, menopause, race/ethnicity, marital status, education) are important in terms of predicting the need for total joint replacement but have no role in evaluating the efficacy of a clinical intervention. As discussed in a previous ESCEO 
meeting, the presence of abnormal biomechanics or severe malalignment may indicate the use of a certain class of treatment and predict the lack of efficacy of interventions (e.g., weight loss or doxycycline [29]) on structural progression. Inflammation (presence of effusion and/or softtissue swelling) is a predictor of progression to total joint replacement [30]. Some inconsistent data suggest that the presence of effusion is predictive of the efficacy of an intraarticular corticosteroid joint injection [31]. Polyarticular joint involvement is a predictor of OA progression, not necessarily of the clinical response.

\subsection{Biochemical Biomarkers}

A systematic review of serum and urine biomarkers in knee and hip OA provides an overview of publications on biochemical markers performance [32]. Data were categorized according to the five categories of the BIPED classification proposed by the National Institutes of Health (NIH)-funded OA Biomarkers Network [33]: Burden of disease, Investigative, Prognosis, Efficacy of intervention, and Diagnostic. A total of 26 biochemical markers were identified, 15 concerned collagen metabolism, eight related to collagen type Il degradation (CTX-II, HELIX-11, C2C, Coll2-1, Coll2-1 NO(2), TIINE) and synthesis (PIIANP, PIICP), five to collagen type 1 degradation (NTX-1, CTX-1, ICTP) and synthesis (PICP, PINP), one to collagen type I and 11 degradation $(\mathrm{C} 1,2 \mathrm{C})$, and one to collagen type III synthesis (PIIINP). It was concluded that none of the current biochemical markers, of cartilage, bone, or synovial metabolism, is sufficiently discriminating to aid diagnosis and prognosis in individual patients with $\mathrm{OA}$, to facilitate the design of clinical trials or to act as a surrogate outcome used as an additional secondary endpoint. This is a view shared by the ESCEO working meeting on biomarkers [7]. However, it must be taken into account that the current perceived limitations in the use of biomarkers stems in part from the limited tools available for their qualification, i.e., the means of establishing the context in which they are validated.

A nested case-control study of progressive knee OA within the OsteoArthritis Initiative has been initiated by the Foundation of NIH OA Biomarkers Consortium to determine the biomarkers with optimal predictive validity and responsiveness for progression of knee OA [5]. The 12 assessed biochemical markers are related to cartilage degradation (CTX-II, C1, 2C, C2C, Coll2-1NO2 in urine and COMP, C1, 2C, C2C, CPII, PIIANP, CS846, MMP-3 in serum), bone resorption [serum and urine NTX-I and CTX-I), and synovitis (serum hyaluronic acid (HA)]. The next step should be the qualification of the most promising markers, either prospectively or using data from completed clinical trials.

\subsection{Genetic and Epigenetic Markers}

New technologies are now available to investigate the link between cellular mechanisms and the disease phenotypes, including the omics approach (genomomics, transcriptomics, proteomics, epigenomics, microbiomics, metabolomics) the exposome (the combined exposures from all sources received by a person during life), new non-hierarchical approaches to phenotyping complex disease (e.g., cluster analyses), applications of informatics to interrogate large data-sets from biological collections, clinical trials, and linked population-based case records and prescribing practice.

A genetic component to $\mathrm{OA}$ in most of the large joints has been established [34]. Recent developments of the identification of the susceptibility genes for OA by using different approaches including genome-wide association studies, family-based studies, and extreme OA phenotypes have been recently reviewed, showing rapid progress in the knowledge of genetics of OA. Despite numerous efforts made on human genetic studies worldwide, only a few numbers of loci have been associated with OA. This might be attributed to several factors such as insufficient sample sizes and disease heterogeneity that might result from different underlying causes, both genetic and environmental, depending on which joints are affected [35]. OA is a complex polygenic disease that lacks any large-effect susceptibility loci. Instead, OA susceptibility alleles individually contribute only modestly to the overall disease risk, making their identification challenging. Although the success of genetic studies is still limited, recent data suggest that epigenetic mechanisms may be heavily mechanistically involved [36].

\subsection{Genetic Biomarkers}

In a recent meta-analysis of nine genome-wide association studies using Human Genome Epidemiology (HuGE) navigator in patients with knee and hip OA, only 2 of the 199 candidate genes considered were shown to be associated with hip OA: COL11A1 (collagen, type XI, alpha 1) and vascular endothelial growth factor (VEGF, signal found only in men) [37].

Other examples are:

- That genetic modification of the SMAD3 gene was found to be associated with hip and knee OA in European populations [38], and also with hand and knee OA in a northeast Chinese population [39];

- TRPV1 (transient receptor potential cation channel, subfamily V, member 3) and COMT (catechol-Omethyltransferase) variants are involved in the perception of pain in knee OA [40, 41]; 
- FTO (fat mass and obesity-associated) signal is robustly associated with BMI, and showed evidence of association with OA underpinning the known epidemiological link between BMI and OA [42];

- The IL1RN (interleukin-1 receptor antagonist) C-T-A haplotype may have a role in severe knee OA which is consistent with the possible role of IL-1 as a regulator of cartilage degradation [43];

- COL11A1, which could play a role in erosion of joints cartilage in OA, is a strong candidate gene for OA [44];

- GDF5 (Growth and differentiation factor 5), to promote the development, maintenance, and repair of joint tissues is associated with OA of the knee with genomewide statistical significance [45];

- The Semaphorin 4D (Sema4D) gene region may play a role in the etiology of acetabular dysplasia [46], a multifactorial disease that has been shown to increase the risk of OA [47].

Moreover, studies are being set up to unravel the genetic causes of OA, including a 5-year study by Arthritis Research UK focusing on rare genetic variants that lead to the development of OA, and looking at how they interact with environmental factors to increase the risk of developing OA.

\subsection{Epigenetic Biomarkers}

Although limited compared with genetic studies, epigenetic studies suggest that post-transcriptional regulation of gene expression might play a substantial role in OA progression. In particular, microRNA-mediated mechanisms have been found to be differentially expressed in normal and OA cartilage $[48,49]$ and offer potential diagnostic and therapeutic strategies, which need to be further investigated.

\section{Frailty and OA}

\subsection{The Frailty Phenotype}

In patients with $\mathrm{OA}$, the presence of coexistent chronic conditions, particularly heart disease, pulmonary disease, and obesity, increase markedly the likelihood of subsequent disability [50]. Consequently, there is a need to assess comorbidity, which is one of the main risk factors for frailty.

There is a growing consensus that comorbidity, disability, and frailty are distinct clinical entities with distinct prevention and therapeutic issues, important overlap, and a causal relationship [51]. Frailty is generally described as "a multidimensional syndrome characterized by decreased reserve and diminished resistance to stressors" [52]. There are two main ways of defining frailty: the Cardiovascular Health Study index [53] defines frailty as a clinical syndrome in which three or more of the following phenotype indicators are present: unintentional weight loss, self-reported exhaustion, weakness, slow walking speed, and low physical activity. Alternatively, the Geriatric Status Scale [54] combines aspects of cognitive and functional performance to describe various degrees of frailty from "robust" to "severely frail" to predict death or need for institutional care.

In the SHARE study, a multidisciplinary European Union Research project, the prevalence of frailty and prefrailty in 18,227 randomly selected community-dwelling men and women aged 50 years and older reached around 10 and $50 \%$, respectively [55]. In four large prospective cohort studies, frailty was shown to be associated with all negative outcomes (falls, disability, hospitalization, home care admission, mortality) [56]. A study based on data from cohorts of community-dwelling persons aged 65 years or older showed that adding frailty markers to age, sex, and chronic diseases provided an increase in the patient-level prediction of disability [57]. The increase was modest but worthwhile because frailty is potentially a reversible condition when effective interventions are put in place [58]. There is thus an urgent need to identify pre-frail and frail older adults to prevent rapid disability in our OA aging population [59].

\subsection{Association of OA with Frailty Status}

Comorbidity and frailty status affect the treatment of OA in many different ways, including the ability to adhere to exercise, polypharmacy (with the potential for drug-drug interactions and increased risk of adverse events), medical contraindications, worsening of quality of life, and mood disorders such as depression and anxiety that can accompany any chronic condition. Several studies have recently examined the association of OA with frailty, all suggesting that the frailty status should be assessed when considering treatment of OA, as it may be essential in targeting therapeutic interventions. The EPOSA study (2942 patients aged 65-85 years) showed a significant association between clinical OA at any site and frailty status (preliminary results). In a cohort of 4130 men aged over 65 years, hip OA and total hip replacement were found to be associated with greater frailty, suggesting that interventions to reduce frailty should be evaluated in these patients [60]. People with lower extremity OA had a two to five times increased incidence of falls than age-matched healthy controls [61]. The mechanisms underlying this increased risk are not clear but could involve poor physical performance, loss of proprioception, and impaired balance [62, 63]. Another study showed that clinical frailty significantly 
predicted mortality in subjects with OA. Thus, clinical frailty may be considered a new prognostic factor to identify subjects with OA at high risk of mortality [64].

The intervention will need to be targeted to the exact phenotype of the frailty identified.

\subsection{Ongoing Projects with a Strong Potential for Synergies and Complementarities in the Fields of Frailty and $\mathrm{OA}$}

There are several international projects in progress that have the potential to further advance the importance and understanding of the interaction between OA and frailty. The Innovative Medicines Initiative (IMI), a joint undertaking between the European Union and the pharmaceutical industry association EFPIA, is supporting the SPRINTTPROJECT (Sarcopenia and Physical Frailty in older people: multi-component treatment). The IMI is additionally funding the APPROACH (Applied PublicPrivate Research enabling OsteoArthritis Clinical Headway) project, which will enhance the collective knowledge base around OA biomarkers and disease as well as build stronger collaborations among academic and industrial groups to enable effective therapeutic development. The "European Innovation Partnership on Active and Healthy Ageing" project promotes systematic routine screening for pre-frailty stages in at-risk patients and older people using the Mmultidimensional Prognostic Index [65]. This index is a multidimensional tool measuring frailty and prognostic indicators in health-related outcomes.

\section{Conclusions}

Our increasing knowledge of disease and drug mechanisms has led to the understanding that the heterogeneity in disease expression and response to therapy is the result of differing underlying pathological mechanisms, as well as other individual patient features that influence treatment efficacy and tolerability. Considering the heterogeneous nature of OA, the very large number of affected individuals, and the need for new efficient and safe treatments, OA appears as an optimal candidate for personalized healthcare [66].

The ultimate aim of these discussions of the ESCEO working group was to explore the value of (bio)markers of different sources in defining different phenotypes of patients with OA. The characterization of the OA patient phenotype could be used to guide clinical decision making. Optimizing the selection of patients for whom an intervention with a DMOAD could prevent the development of OA would assist the emergence of personalized or precision medicine for these patients.
A research agenda for the identification of patient phenotypes was proposed (Table 1). This approach should lead to the definition of a risk score, allowing the categorization of patients according to their risk for structural or clinical progression. This should include all possible predictors, possibly including an aggregate of different (bio)markers.

As part of this agenda, it was felt important to recommend a core data set that should be collected in future studies of OA that assesses the natural history of the disease and the response to treatment. This will allow more standardized assessment and collation of cohorts and databases in the future. Following the meeting, the working group produced a recommended list of core data, presented in Table 2, which was based on the evidence provided at the meeting and individual expert opinion.

Profiles of patients may be defined according to their level of pain, functional limitation, and presence of coexistent chronic conditions including frailty status. Moreover, converging data argue for an MRI-based diagnosis in clinical practice to help physicians identify patients who are more likely to benefit from treatment. More research is required, however, to validate these preliminary findings and to correlate improvements with hard clinical endpoints.

Among potential biochemical biomarkers, despite active research and numerous candidates, none is sufficiently validated and recognized for systematic use in drug development of $\mathrm{OA}$ or in clinical practice to identify patients who should be treated. Among current investigations, the Foundation of NIH OA Biomarkers Consortium has established and initiated a process for validation and qualification of the most promising commercially available biomarkers in OA [5]. This approach will support new drug

Table 1 Research agenda for the identification of patient phenotypes

Identify published randomized clinical trials and observational cohorts assessing the efficacy of different class of interventions on clinically relevant outcomes, divided in structural and symptomatic outcomes

Using above data, produce clinical prediction tools to quantify a patient's risk of progression and good outcomes from treatment interventions

With available data, identify phenotypes of patients according to their outcome. Panel of (bio)markers (clinical, biochemical, imaging) should be investigated, rather than individual items

Assess the uniformity of data across clinical trials and cohorts

Proceed to a validation step on a separate validation cohort

Ensure that all new cohorts and trials use the same core dataset to allow easy integration into extant data

Possible limitations:

The availability of the data and of the biological specimens in cohorts

The high heterogeneity in the assessments methods and reporting (e.g., multiple assessment tools for pain), which would require a hierarchical/standardization of criteria 
Table 2 Minimum core data set

Age [67]
BMI [10]
Sex [67, 68]
Racial origin [69]
Occupation [70]
Comorbidities [71]
Menopausal status [72]
Presence of chondrocalcinosis [73]
Baseline pain and function [13, 74 ]
OA pain in other joints [10]
Possibly meniscal extrusion in the target knee [23, 24]
X-ray grade of target joint [10]
OA medication use (analgesia and disease modification)
Previous joint surgery (in particular menisectomy) [75]
Persistent inflammation or effusion [30, 31]
Trauma in the elderly [76, 77]

$B M I$ body mass index, $O A$ osteoarthritis

developments, preventive medicine, and medical diagnostics for OA.

In the future, identification of genes associated with OA may help reveal underlying biological mechanisms that lead to the development of new therapeutic targets or biomarkers for early detection and risk stratification. Considerable efforts are being made to identify genetic and epigenetic factors involved in OA that could be used as potential stratifiers, but results are still limited. However, with new technologies, diagnosis will increasingly focus on integrating information for multiple sources, not only genomics and other omics technologies but also environmental and lifestyle data.

Acknowledgments This meeting was founded by the European Society for Clinical and Economics Aspect of Osteoporosis and Osteoarthritis, a not for profit organization from Belgium.

Conflicts of interest Pr. Arden has received consultancy fees from Flexion (pharmanet), Lilly, Merck, Q Med, Roche, Smith \& Nephew, Amgen, GSK, and NiCox, lecture fees from Smith \& Nephew, Amgen, GSK, and NiCox, and grants from Novartis, Pfizer, ScheringPlough, and Servier. Pr. Richette has received lecture fees from Servier, Expanscience, Genevrier, Fidia, Ibsa, and Rottapharm. Pr. Cooper has received consultancy fees from Amgen, GSK, Alliance for Better Bone Health, MSD, Eli Lilly, Pfizer, Novartis, Servier, Merck, Medtronic, and Roche, lecture fees from Amgen, GSK, Alliance for Better Bone Health, MSD, Eli Lilly, Pfizer, Novartis, Servier, Merck, Medtronic, and Roche, and honoraria from Amgen, GSK, Alliance for Better Bone Health, MSD, Eli Lilly, Pfizer, Novartis, Servier, Merck, Medtronic, and Roche. Pr. Bruyère has received personal fees from Bayer, IBSA, Genevrier, MSD, Novartis, Nutraveris, Pfizer, Rottapharm, Servier, SMB, and Theramex, and grants from Bayer, IBSA, Genevrier, MSD, Novartis, Nutraveris, Pfizer, Rottapharm, Servier, SMB, and Theramex. Pr. Abadie, Pr. Brandi, Pr Clerc, Pr. Kanis, Pr. Maggi, Pr. Mautone, F. Petit-Dop, Pr. Tajana, and Dr. Tsouderos declared no conflicts of interests. Pr. Berenbaum has received consultancy fees from Pfizer, Abbvie, Merck
Serono, Nordic Bioscience, Servier, and Jansenn Expanscience, a grant from TRB Chemedica, participation fees from Nordic Bioscience, and lecture fees from Servier, Expanscience, TRB Chemedica, and IBSA. Pr Dennison has received reimbursement for travel expenses from the ESCEO, and lectures fees from Lilly. Pr Devogelaer has received reimbursement for travel expenses from the ESCEO. Pr Hochberg has received reimbursement for travel expenses from the ESCEO, and consultancy fees from Bioiberica, Iroko Pharmaceuticals, Novartis Pharma AG, Pfizer, and Theralogix LLC. Dr D'Hooghe declared no conflicts of interests. Pr Herrero-Beaumont has received reimbursement for travel expenses from the ESCEO. Pr. Laslop has received reimbursement for travel expenses to the meeting for the study. Dr Leblanc is employed by Laboratories Expanscience as a medical director. Pr Pelletier has received reimbursement for travel expenses to the meeting for the study, is a shareholder in Arthrolab Inc., and has received consultancy fees from Abbie, Bioiberica, Elanco, Endocyte, Ferring, Merck \& Co Pfizer, Servier, and TRB Chemedica. Pr. Reiter-Niesert has received reimbursement for travel expenses to the meeting for the study. Pr. Rizzoli has received lecture fees from Amgen, GSK, MSD, Servier, and Danone. Pr. Rovati has received stock options from Rottapharm. Pr. MartelPelletier has received consultancy fees from Abbie, Bioiberica, Endocyte, Merck \& Co, and TRB Chemedica, is a shareholder in Arthrolab Inc, and received reimbursement for travel expenses to the meeting for the study. Pr. Reginster received consultancy fees from Servier, Novartis, Negma, Lilly, Wyeth, Amgen, GlaxoSmithKline, Roche, Merckle, Nycomed, NPS, Theramex, and UCB, lecture fees from Merck Sharp \& Dohme, Lilly, Rottapharm, IBSA, Genevrier, Novartis, Servier, Roche, GlaxoSmithKline, Teijin, Teva, Ebewee Pharma, Zodiac, Analis, Theramex, Nycomed, Novo-Nordisk, and Nolver, and grants from Bristol Myers Squibb, Merck Sharp \& Dohme, Rottapharm, Teva, Lilly, Novartis, Roche, GlaxoSmithKline, Amgen, and Servier.

Author's contribution $\mathrm{OB}$ and JYR organized the meeting. JMP, $\mathrm{MH}, \mathrm{CC}, \mathrm{ED}$, and SM performed the literature review. All authors have taken part in the discussion and meeting and have critically analyzed and approved the final manuscript.

Open Access This article is distributed under the terms of the Creative Commons Attribution-NonCommercial 4.0 International License (http://creativecommons.org/licenses/by-nc/4.0/), which permits any noncommercial use, distribution, and reproduction in any medium, provided you give appropriate credit to the original author(s) and the source, provide a link to the Creative Commons license, and indicate if changes were made.

\section{References}

1. Trusheim MR, Berndt ER, Douglas FL. Stratified medicine: strategic and economic implications of combining drugs and clinical biomarkers. Nat Rev Drug Discov. 2007;6(4):287-93.

2. Hingorani AD, Windt DA, Riley RD, Abrams K, Moons KG, Steyerberg EW, et al. Prognosis research strategy (PROGRESS) 4: stratified medicine research. BMJ. 2013;346:e5793.

3. Ethgen O, Reginster JY. Degenerative musculoskeletal disease. Ann Rheum Dis. 2004;63(1):1-3.

4. Cooper C, Adachi JD, Bardin T, Berenbaum F, Flamion B, Jonsson $\mathrm{H}$, et al. How to define responders in osteoarthritis. Curr Med Res Opin. 2013;29(6):719-29.

5. Hunter DJ, Nevitt M, Losina E, Kraus V. Biomarkers for osteoarthritis: current position and steps towards further validation. Best Pract Res Clin Rheumatol. 2014;28(1):61-71. 
6. Pelletier JP, Cooper C, Peterfy C, Reginster JY, Brandi ML, Bruyere $\mathrm{O}$, et al. What is the predictive value of MRI for the occurrence of knee replacement surgery in knee osteoarthritis? Ann Rheum Dis. 2013;72(10):1594-604.

7. Lotz M, Martel-Pelletier J, Christiansen C, Brandi ML, Bruyere $\mathrm{O}$, Chapurlat R, et al. Value of biomarkers in osteoarthritis: current status and perspectives. Ann Rheum Dis. 2013;72(11): 1756-63.

8. Bruyere O, Cooper C, Arden N, Branco J, Brandi ML, HerreroBeaumont $\mathrm{G}$, et al. Can we identify patients with high risk of osteoarthritis progression who will respond to treatment? A focus on epidemiology and phenotype of osteoarthritis. Drugs Aging. 2015;32(3):179-87.

9. Belo JN, Berger MY, Reijman M, Koes BW, Bierma-Zeinstra SM. Prognostic factors of progression of osteoarthritis of the knee: a systematic review of observational studies. Arthritis Rheum. 2007;57(1):13-26.

10. Lohmander LS, Felson D. Can we identify a 'high risk' patient profile to determine who will experience rapid progression of osteoarthritis? Osteoarthr Cartil. 2004;12 Suppl A:S49-52.

11. Tanamas S, Hanna FS, Cicuttini FM, Wluka AE, Berry P, Urquhart DM. Does knee malalignment increase the risk of development and progression of knee osteoarthritis? A systematic review. Arthritis Rheum. 2009;61(4):459-67.

12. Leyland KM, Hart DJ, Javaid MK, Judge A, Kiran A, Soni A, et al. The natural history of radiographic knee osteoarthritis: a fourteen-year population-based cohort study. Arthritis Rheum. 2012;64(7):2243-51.

13. Soni A, Kiran A, Hart DJ, Leyland KM, Goulston L, Cooper C, et al. Prevalence of reported knee pain over twelve years in a community-based cohort. Arthritis Rheum. 2012;64(4):1145-52.

14. Knoop J, van der Leeden M, Thorstensson CA, Roorda LD, Lems WF, Knol DL, et al. Identification of phenotypes with different clinical outcomes in knee osteoarthritis: data from the Osteoarthritis Initiative. Arthritis Care Res (Hoboken). 2011; 63(11):1535-42.

15. Bruyere O, Cooper C, Pelletier JP, Branco J, Luisa BM, Guillemin $\mathrm{F}$, et al. An algorithm recommendation for the management of knee osteoarthritis in Europe and internationally: a report from a task force of the European Society for Clinical and Economic Aspects of Osteoporosis and Osteoarthritis (ESCEO). Semin Arthritis Rheum. 2014;44(3):253-63.

16. Matthews GL, Hunter DJ. Emerging drugs for osteoarthritis. Expert Opin Emerg Drugs. 2011;16(3):479-91.

17. Lohmander LS, Roos EM. Clinical update: treating osteoarthritis. Lancet. 2007;370(9605):2082-4.

18. Raynauld JP, Martel-Pelletier J, Bias P, Laufer S, Haraoui B, Choquette D, et al. Protective effects of licofelone, a 5-lipoxygenase and cyclo-oxygenase inhibitor, versus naproxen on cartilage loss in knee osteoarthritis: a first multicentre clinical trial using quantitative MRI. Ann Rheum Dis. 2009;68(6):938-47.

19. Wildi LM, Raynauld JP, Martel-Pelletier J, Beaulieu A, Bessette L, Morin F, et al. Chondroitin sulphate reduces both cartilage volume loss and bone marrow lesions in knee osteoarthritis patients starting as early as 6 months after initiation of therapy: a randomised, double-blind, placebo-controlled pilot study using MRI. Ann Rheum Dis. 2011;70(6):982-9.

20. Pelletier JP, Roubille C, Raynauld JP, Abram F, Dorais M, Delorme $\mathrm{P}$, et al. Disease-modifying effect of strontium ranelate in a subset of patients from the phase III knee osteoarthritis study SEKOIA using quantitative MRI: reduction in bone marrow lesions protects against cartilage loss. Ann Rheum Dis. 2015; 74(2):422-9.

21. Lohmander LS, Hellot S, Dreher D, Krantz EF, Kruger DS, Guermazi A, et al. Intraarticular sprifermin (recombinant human fibroblast growth factor 18) in knee osteoarthritis: a randomized, double-blind, placebo-controlled trial. Arthritis Rheumatol. 2014;66(7):1820-31.

22. Martel-Pelletier J, Roubille C, Abram F, Hochberg MC, Dorais M, Delorme P, et al. First-line analysis of the effects of treatment on progression of structural changes in knee osteoarthritis over 24 months: data from the osteoarthritis initiative progression cohort. Ann Rheum Dis. 2015;74(3):547-56.

23. Berthiaume MJ, Raynauld JP, Martel-Pelletier J, Labonte F, Beaudoin G, Bloch DA, et al. Meniscal tear and extrusion are strongly associated with progression of symptomatic knee osteoarthritis as assessed by quantitative magnetic resonance imaging. Ann Rheum Dis. 2005;64(4):556-63.

24. Pelletier JP, Raynauld JP, Berthiaume MJ, Abram F, Choquette $\mathrm{D}$, Haraoui B, et al. Risk factors associated with the loss of cartilage volume on weight-bearing areas in knee osteoarthritis patients assessed by quantitative magnetic resonance imaging: a longitudinal study. Arthritis Res Ther. 2007;9(4):R74.

25. Roubille C, Martel-Pelletier J, Abram F, Dorais M, Delorme P, Raynauld JP, et al. Insight into the role of meniscal extrusion and bone marrow lesions in knee osteoarthritis progression and their impact on response to strontium ranelate treatment in a subset of patients from the SEKOIA study. Ann Rheum Dis. 2014; 73(Suppl 2):745.

26. Pelletier JP, Roubille C, Abram F, Dorais M, Delorme P, Raynauld JP, et al. The effects of treatment on disease symptoms and progression of structural changes in knee osteoarthritis participants from the osteoarthritis initiative progression cohort. Arthritis Rheum. 2014;66(Suppl 11):S979.

27. Roubille C, Raynauld JP, Abram F, Paiement P, Dorais M, Delorme $\mathrm{P}$, et al. The presence of meniscal lesions is a strong predictor of neuropathic pain in symptomatic knee osteoarthritis: a cross sectional pilot study. Arthritis Res Ther. 2014;16(6): 507-14.

28. Roemer FW, Felson DT, Wang K, Crema MD, Neogi T, Zhang Y, et al. Co-localisation of non-cartilaginous articular pathology increases risk of cartilage loss in the tibiofemoral joint: the MOST study. Ann Rheum Dis. 2013;72(6):942-8.

29. Mazzuca SA, Brandt KD, Chakr R, Lane KA. Varus malalignment negates the structure-modifying benefits of doxycycline in obese women with knee osteoarthritis. Osteoarthr Cartil. 2010;18(8):1008-11.

30. Conaghan PG, D'Agostino MA, Le BM, Baron G, Schmidely N, Wakefield $\mathrm{R}$, et al. Clinical and ultrasonographic predictors of joint replacement for knee osteoarthritis: results from a large, 3-year, prospective EULAR study. Ann Rheum Dis. 2010;69(4):644-7.

31. Maricar N, Callaghan MJ, Felson DT. O'neill TW. Predictors of response to intra-articular steroid injections in knee osteoarthritis: a systematic review. Rheumatology (Oxford). 2013;52(6):1022-32.

32. Van Spil WE, DeGroot J, Lems WF, Oostveen JC, Lafeber FP. Serum and urinary biochemical markers for knee and hip-osteoarthritis: a systematic review applying the consensus BIPED criteria. Osteoarthr Cartil. 2010;18(5):605-12.

33. Bauer DC, Hunter DJ, Abramson SB, Attur M, Corr M, Felson D, et al. Classification of osteoarthritis biomarkers: a proposed approach. Osteoarthr Cartil. 2006;14(8):723-7.

34. Spector TD, Cicuttini F, Baker J, Loughlin J, Hart D. Genetic influences on osteoarthritis in women: a twin study. BMJ. 1996;312(7036):940-3.

35. Valdes AM, McWilliams D, Arden NK, Doherty SA, Wheeler M, Muir KR, et al. Involvement of different risk factors in clinically severe large joint osteoarthritis according to the presence of hand interphalangeal nodes. Arthritis Rheum. 2010;62(9):2688-95.

36. Barter MJ, Young DA. Epigenetic mechanisms and non-coding RNAs in osteoarthritis. Curr Rheumatol Rep. 2013;15(9):353. 
37. Rodriguez-Fontenla C, Calaza M, Evangelou E, Valdes AM, Arden N, Blanco FJ, et al. Assessment of osteoarthritis candidate genes in a meta-analysis of nine genome-wide association studies. Arthritis Rheumatol. 2014;66(4):940-9.

38. Valdes AM, Spector TD, Tamm A, Kisand K, Doherty SA, Dennison EM, et al. Genetic variation in the SMAD3 gene is associated with hip and knee osteoarthritis. Arthritis Rheum. 2010;62(8):2347-52.

39. Liying J, Yuchun T, Youcheng W, Yingchen W, Chunyu J, Yanling Y, et al. A SMAD3 gene polymorphism is related with osteoarthritis in a Northeast Chinese population. Rheumatol Int. 2013;33(7):1763-8.

40. Valdes AM, De WG, Doherty SA, Lories RJ, Vaughn FL, Laslett $\mathrm{LL}$, et al. The Ile585Val TRPV1 variant is involved in risk of painful knee osteoarthritis. Ann Rheum Dis. 2011;70(9):1556-61.

41. Neogi T, Soni A, Doherty SA, Laslett LL, Maciewicz RA, Hart DJ, et al. Contribution of the COMT Val158Met variant to symptomatic knee osteoarthritis. Ann Rheum Dis. 2014;73(1):315-7.

42. Elliott KS, Chapman K, Day-Williams A, Panoutsopoulou K, Southam L, Lindgren CM, et al. Evaluation of the genetic overlap between osteoarthritis with body mass index and height using genome-wide association scan data. Ann Rheum Dis. 2013;72(6):935-41.

43. Kerkhof HJ, Doherty M, Arden NK, Abramson SB, Attur M, Bos $\mathrm{SD}$, et al. Large-scale meta-analysis of interleukin-1 beta and interleukin-1 receptor antagonist polymorphisms on risk of radiographic hip and knee osteoarthritis and severity of knee osteoarthritis. Osteoarthr Cartil. 2011;19(3):265-71.

44. Raine EV, Dodd AW, Reynard LN, Loughlin J. Allelic expression analysis of the osteoarthritis susceptibility gene COL11A1 in human joint tissues. BMC Musculoskelet Disord. 2013;14:85.

45. Valdes AM, Evangelou E, Kerkhof HJ, Tamm A, Doherty SA, Kisand K, et al. The GDF5 rs143383 polymorphism is associated with osteoarthritis of the knee with genome-wide statistical significance. Ann Rheum Dis. 2011;70(5):873-5.

46. Sekimoto T, Ishii M, Emi M, Kurogi S, Funamoto T, Hamada H, et al. Segmental copy number loss in the region of Semaphorin 4D gene in patients with acetabular dysplasia. J Orthop Res. 2013;31(6):957-61.

47. McWilliams DF, Doherty SA, Jenkins WD, Maciewicz RA, Muir $\mathrm{KR}$, Zhang W, et al. Mild acetabular dysplasia and risk of osteoarthritis of the hip: a case-control study. Ann Rheum Dis. 2010;69(10): 1774-8.

48. Diaz-Prado S, Cicione C, Muinos-Lopez E, Hermida-Gomez T, Oreiro N, Fernandez-Lopez C, et al. Characterization of microRNA expression profiles in normal and osteoarthritic human chondrocytes. BMC Musculoskelet Disord. 2012;13:144.

49. Iliopoulos D, Malizos KN, Oikonomou P, Tsezou A. Integrative microRNA and proteomic approaches identify novel osteoarthritis genes and their collaborative metabolic and inflammatory networks. PLoS One. 2008;3(11):e3740.

50. Ettinger WH, Davis MA, Neuhaus JM, Mallon KP. Long-term physical functioning in persons with knee osteoarthritis from NHANES. I: effects of comorbid medical conditions. J Clin Epidemiol. 1994;47(7):809-15.

51. Fried LP, Ferrucci L, Darer J, Williamson JD, Anderson G. Untangling the concepts of disability, frailty, and comorbidity: implications for improved targeting and care. J Gerontol A Biol Sci Med Sci. 2004;59(3):255-63.

52. Rodriguez-Manas L, Feart C, Mann G, Vina J, Chatterji S, Chodzko-Zajko W, et al. Searching for an operational definition of frailty: a Delphi method based consensus statement: the frailty operative definition-consensus conference project. J Gerontol A Biol Sci Med Sci. 2013;68(1):62-7.

53. Fried LP, Tangen CM, Walston J, Newman AB, Hirsch C, Gottdiener J, et al. Frailty in older adults: evidence for a phenotype. J Gerontol A Biol Sci Med Sci. 2001;56(3):M146-56.
54. Rockwood K, Song X, MacKnight C, Bergman H, Hogan DB, McDowell I, et al. A global clinical measure of fitness and frailty in elderly people. CMAJ. 2005;173(5):489-95.

55. Santos-Eggimann B, Cuenoud P, Spagnoli J, Junod J. Prevalence of frailty in middle-aged and older community-dwelling Europeans living in 10 countries. J Gerontol A Biol Sci Med Sci. 2009;64(6):675-81.

56. Clegg A, Young J, Iliffe S, Rikkert MO, Rockwood K. Frailty in elderly people. Lancet. 2013;381(9868):752-62.

57. Sourial N, Bergman H, Karunananthan S, Wolfson C, Payette H, Gutierrez-Robledo LM, et al. Implementing frailty into clinical practice: a cautionary tale. J Gerontol A Biol Sci Med Sci. 2013;68(12):1505-11.

58. Cesari M. The European Union Geriatric Medicine Society (EUGMS) working group on "frailty in older persons". J Frailty Aging 2013;118-20.

59. Vellas B, Cestac P, Moley JE. Implementing frailty into clinical practice: we cannot wait. J Nutr Health Aging. 2012;16(7):599-600.

60. Wise BL, Parimi N, Zhang Y, Cawthon PM, Barrett-Connor E, Ensrud KE, et al. Frailty and hip osteoarthritis in men in the MrOS cohort. J Gerontol A Biol Sci Med Sci. 2014;69(5):602-8.

61. Hoops ML, Rosenblatt NJ, Hurt CP, Crenshaw J, Grabiner MD. Does lower extremity osteoarthritis exacerbate risk factors for falls in older adults? Womens Health (Lond Engl). 2012;8(6):685-96.

62. Sharma L, Pai YC, Holtkamp K, Rymer WZ. Is knee joint proprioception worse in the arthritic knee versus the unaffected knee in unilateral knee osteoarthritis? Arthritis Rheum. 1997;40(8):1518-25.

63. Edwards MH, van der Pas S, Denkinger MD, Parsons C, Jameson KA, Schaap L, et al. Relationships between physical performance and knee and hip osteoarthritis: findings from the European Project on Osteoarthritis (EPOSA). Age Ageing. 2014;43(6): 806-13.

64. Cacciatore F, Della-Morte D, Basile C, Mazzella F, Mastrobuoni C, Salsano E, et al. Long-term mortality in frail elderly subjects with osteoarthritis. Rheumatology (Oxford). 2014;53(2):293-9.

65. Pilotto A, Ferrucci L, Franceschi M, D’Ambrosio LP, Scarcelli C, Cascavilla L, et al. Development and validation of a multidimensional prognostic index for one-year mortality from comprehensive geriatric assessment in hospitalized older patients. Rejuvenation Res. 2008;11(1):151-61.

66. Karsdal MA, Christiansen C, Ladel C, Henriksen K, Kraus VB, Bay-Jensen AC. Osteoarthritis: a case for personalized health care? Osteoarthr Cartil. 2014;22(1):7-16.

67. Prieto-Alhambra D, Judge A, Javaid MK, Cooper C, Diez-Perez A, Arden NK. Incidence and risk factors for clinically diagnosed knee, hip and hand osteoarthritis: influences of age, gender and osteoarthritis affecting other joints. Ann Rheum Dis. 2014; 73(9):1659-64

68. Dougados M, Gueguen A, Nguyen M, Berdah L, Lequesne M, Mazieres B, et al. Radiological progression of hip osteoarthritis: definition, risk factors and correlations with clinical status. Ann Rheum Dis. 1996;55(6):356-62.

69. Zhang Y, Jordan JM. Epidemiology of osteoarthritis. Clin Geriatr Med. 2010;26(3):355-69.

70. McWilliams DF, Leeb BF, Muthuri SG, Doherty M, Zhang W. Occupational risk factors for osteoarthritis of the knee: a metaanalysis. Osteoarthr Cartil. 2011;19(7):829-39.

71. van Dijk GM, Veenhof C, Spreeuwenberg P, Coene N, Burger BJ, van Schaardenburg SD, et al. Prognosis of limitations in activities in osteoarthritis of the hip or knee: a 3-year cohort study. Arch Phys Med Rehabil. 2010;91(1):58-66.

72. Wluka AE, Cicuttini FM, Spector TD. Menopause, oestrogens and arthritis. Maturitas. 2000;35(3):183-99.

73. Abhishek A, Doherty S, Maciewicz R, Muir K, Zhang W, Doherty $\mathrm{M}$. Evidence of a systemic predisposition to chondrocalcinosis and association between chondrocalcinosis and 
osteoarthritis at distant joints: a cross-sectional study. Arthritis Care Res (Hoboken). 2013;65(7):1052-8.

74. Cooper C, Snow S, McAlindon TE, Kellingray S, Stuart B, Coggon $\mathrm{D}$, et al. Risk factors for the incidence and progression of radiographic knee osteoarthritis. Arthritis Rheum. 2000;43(5):995-1000.

75. Roos H, Lauren M, Adalberth T, Roos EM, Jonsson K, Lohmander LS. Knee osteoarthritis after meniscectomy: prevalence of radiographic changes after twenty-one years, compared with matched controls. Arthritis Rheum. 1998;41(4):687-93.
76. Cooper C, Inskip H, Croft P, Campbell L, Smith G, McLaren M, et al. Individual risk factors for hip osteoarthritis: obesity, hip injury, and physical activity. Am J Epidemiol. 1998;147(6):516-22.

77. Richmond SA, Fukuchi RK, Ezzat A, Schneider K, Schneider G, Emery CA. Are joint injury, sport activity, physical activity, obesity, or occupational activities predictors for osteoarthritis? A systematic review. J Orthop Sports Phys Ther. 2013;43(8):B515-9. 